\title{
Applying User Interface Design Process Model for a Mobile Community Project for the Deaf
}

\author{
Chui Yin Wong, Chee Weng Khong and Harold Thwaites \\ Multimedia University, Malaysia
}

\begin{abstract}
The advancement of mobile communication technologies has created a new form of social network for communities to communicate and share similar interests via the mobile platform. Given the fact that Malaysia is a fastpaced developing nation with a relatively low computerInternet penetration, and high mobile network growth, there are desirable needs for disabled communities to leverage a mobile social network to enhance relationships for better communication in their daily lives. We designed DHearT, a mobile social network for hearing impaired users in Malaysia to bridge the communication gap between the deaf and hearing groups. This paper illustrates and discusses the user interface design process model with its design methods and human factors techniques for a mobile community project for the deaf, namely DHearT.
\end{abstract}

Index Terms-online community, mobile, deaf, design process model, design methods, human factors techniques and interface design.

\section{INTRODUCTION}

The advent of mobile technologies is creating a digital paradigm shift, especially in the way we communicate. Having a mobile device, such as a mobile phone, has become a necessity for communication and a commodity for everyone while on the move. We, therefore, can find an increasing trend where online communities are beginning to appear on cellular networks, reaching out to the greater masses (e.g. mobile devices, mobile social networks) that are constantly on the move. This leaves a lot of room for potential research work among humancomputer interaction research communities. However, there are limited studies conducted on mobile communities for the handicapped and disabled users, particularly for the deaf community in a developing nation like Malaysia.

Malaysia is a fast-paced developing nation, which is relatively low in computer-Internet penetration but high in mobile-network growth. For an estimated 24 million population in Malaysia, the adoption and use of mobile phones (80.8 per 100 inhabitants) has increased exponentially and surpassed the subscription rate of Internet dial-up (14.3 per 100 inhabitants), and fixed-line telephones (15.9 per 100 inhabitants) for the third Quarter of 2007 [1]. The population of the hearing impaired and deaf is estimated at 32,000 in Malaysia, and many rely on mobile networks to communicate with their friends and families whilst on the move. It is highly desirable for the deaf community to have a localized and alluring social channel on mobile networks that caters to their niche interest. Interactions by the deaf society through such cellular and wireless networks may well be for the exchange of information, sharing special interests and hobbies, sharing experiences, live-streaming special moments, gossiping, providing medical advice, moral support, exchanging views, and also exchanging experiences in life.

Given the communication barriers between the deaf and the hearing communities, we have developed a prototype of a digital community for the deaf via a mobile network, namely DHearT. Our aim is to bridge the communication gap between the deaf and the hearing communities while on the move. This paper illustrates the user interface design process model and design methods that we applied and adopted in the DHearT mobile community design project.

\section{DeAF COMMUnities IN MALAysia}

\section{A. Deaf Communities in Malaysia}

According to the local Social Welfare Department in 2005, there were approximately 32,000 deaf people who officially registered, out of the country's population of approximately 23 million. We believe the actual deaf population is far greater than the abovementioned figure. Although the number may seem small, the needs and demands using ICT on mobile networks through improvements on mobile technology supported by phone manufacturers, service providers and networking technologies are essential to improve their quality of everyday lives and among their communities [2].

The Malaysian Council for Rehabilitation [3] stated that disability issues have been identified, but the action plans relating to its recommendations are yet to be fully implemented to achieve the social equality targeted for the disabled community. The current state of affairs are to convene all relevant NGO's (Non-Governmental Organizations) efforts in the country to highlight the awareness and to integrate social welfare benefits (healthcare, employment, education) into legislative policies. However, the policy-making process seems to be delayed in the last decade. This highlights a need for an alternative means of enhancing social and communal interactions positively.

There are, of course, various categories of deafness. According to the W3C [4], hearing impairment is categorized as 'deafness' and 'loss of hearing'. Deafness involves a substantial uncorrectable impairment of hearing in both ears. Some deaf individuals 'first language' is a sign language, and they may or may not read a written language fluently, or speak clearly. Hard of hearing means a person with a mild to moderate hearing 
impairment. In this paper, we refer the hard of hearing, hearing impaired person, those who are born deaf and the medically diagnosed deaf person as “deaf”. This paper does not refer to the terms of "deaf community" and "deaf culture", which usually indicates the person(s) who is/are culturally deaf as opposed to the person(s) who is/are diagnosed deaf from a medical, audiological or pathological viewpoint [5].

The main communication mode for the deaf society in Malaysia is sign language. "Total Communication" education was introduced in local schools for the deaf population since 1978. Total Communication emphasizes all forms of appropriate approaches to oral, manual (sign) and auditory training. The primary objective is to equip the deaf community with the necessary skills to communicate with each other and basic interaction skill sets. It includes oralism, lip-reading manually coded local Malay Language (Bahasa Malaysia Kod Tangan $B M K T)$, natural signs and cues in speech. The BMKT is a coded manual with its structure like a spoken language, which adheres to the basic principles and grammar of Bahasa Malaysia (the national language of Malaysia), Malaysian Sign Language (Bahasa Isyarat Malaysia $B I M)$ is the official structured sign language [5].

In general, the education and communication for the local deaf community are mainly in Bahasa Malaysia and BIM (Malaysian Sign Language). The Malaysian deaf community was exposed to English education later than the hearing people during their school time. Most of the local deaf community receives special education in their early age. The good students usually will join the hearing students during high school whereas the average groups will continue their education in the special education schools.

The deaf social and cultural values are unique. Padden and Humphries [6] defined culture as a set of learned behaviors of a group of people who have their own language, values, rules of behaviors, and traditions. It is a culture that usually gives people their sense of identity, whether at an individual or group level. Likewise, the deaf culture also shares arts, humor, common stories, and gossips to others in the community. The deaf community all over the world view themselves as belonging to a linguistic minority with its own culture. The deaf community considers "sign" as their own mother tongue and main language. The deaf culture has its own history, shared values, social norms, customs and technology which are transferred from generation to generation.

Deaf people have had a sense of kinship and unity for a long time. In the deaf culture, they often take it upon themselves to share information, any at all, with the rest of their community. The information shared generally tends to be deeper, appreciated and slightly more intrinsic than that shared in the hearing culture [7]. The sharing indicates that there is a depth of relationship, the setting, and the time for sharing from the details of sharing of information than is common to the hearing culture.

It is inevitable and natural that the command of the English language for the local deaf community is less efficient, less fluent and lower in standards compared to the normal hearing society. Somehow this social disparity is clearly shown in the daily communication between the hearing and the deaf through either traditional communication channels (e.g. pen and pencil with paper) or online communication tools (online chats, e-mails, and so forth).

\section{ONLINE COMMUNITIES FOR THE DEAF}

\section{A. Existing Online Communities for the Deaf}

Online communities can explore new forms of social networking and experience the changing concepts of community as social groups develop within a mixed realm of innovative technologies, creative systems and ubiquitous services. It changes the nature of personal identity, social organization and the connections of realworld communities. Users produce, distribute and consume creative content. They communicate in verbal and non-verbal ways, implicitly and explicitly input selected versions of their everyday lives on digital display. This generates a dynamic and eventful social environment. Furthermore, the communities are wellconnected via wireless devices and Internet services. Societies will inevitably formulate new era of communities, who are constantly able to access to the latest information and services. The online community can provide new modes and shape the ways people work, learn, live and become any group of people who use Internet technologies to communicate with each other. It depends on whether one takes a social perspective or a technology perspective; online communities tend to be named by the activity and people that serve or the technology that supports them.

Generally, the current popular online communities that attract the most frequent online traffic and users are Friendster, MySpace and Facebook. These sites are popular among young adults and the groupings tend to be sharing general interest rather than specific interests. These online community sites share similar features such as user profiles, blogs, friend lists, sharing photos, forums and groups.

There are other online communities that are growing exponentially, for instance, LinkedIn Community is specifically targeted for professional communities with the highlight on relationship-building and networking purposes. Although those sites generally attract thousands of people from all over the world to share common interests and to foster friendships, it is uncertain as to whether or not such a site produces relevant content, and whether it is focused to the needs and interest of the deaf community. The following are some existing online community sites that cater to the needs of the deaf community.

\section{B. Examples of Online Communities for the Deaf}

Deaf Online (Fig.1) is a forum that has an estimated 11 million deaf people around the world joining in.

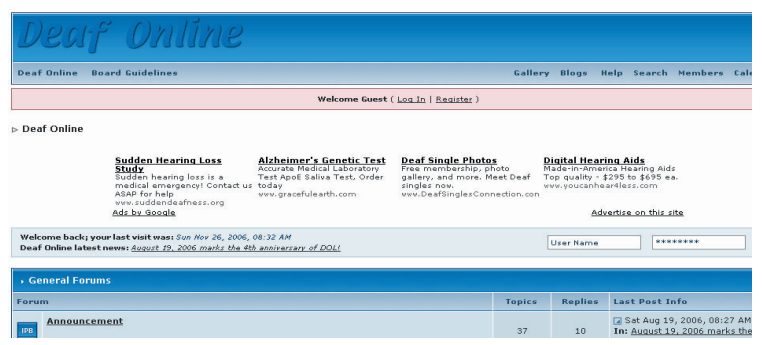

Figure 1. Deaf Online 
Basically, the deaf communities discuss issues related to their hearing impairment, and share their interests on assistive technologies, deaf education, hobbies, book discussions, sports, and so forth [8].

All Deaf Forum (Fig. 2) is a popular online community with thousands of deaf and hard of hearing people from all over the world. A new member is required to join and register themselves in order to access the online forum. The forum attracts both the deaf and also the hearing community to contribute and to share common interest, issues and hobbies [9].

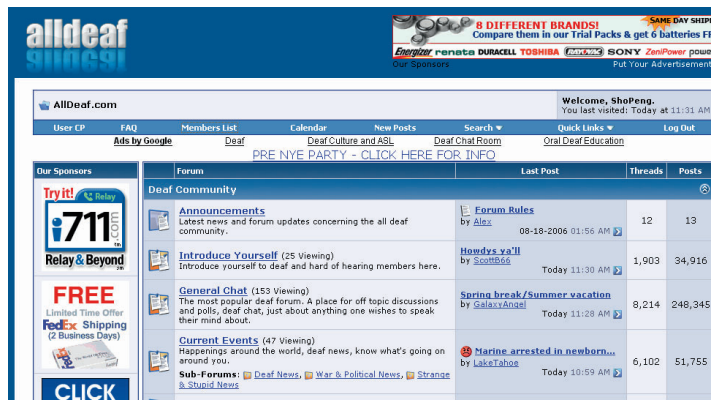

Figure 2. All Deaf Forum

Tag Deaf (Fig. 3) is a free, social network for the deaf on the web. It offers free registration and helps the deaf, hard of hearing and any other hearing people get connected from all over the world. Tag Deaf provides MySpace-like atmosphere where the deaf community can network and socialize among the community [10]. The site offers a multitude of features with free registration, including picture sharing, blogs, videos and games, forums, customized profiles, classifieds, instant messaging, groups and polls. The simple design and feature set are reminiscent of a social network such as MySpace, but the focus is towards the hearing impaired community worldwide. Members of the social network also have the ability to invite others to the site, and search for or browse members with similar interests to help them connecting to each other.

Deaf Singles Connection (Fig. 4) is an online, social website that is designed for the deaf to find their potential lifelong and future partners, or to meet their match. It is an online community dedicated to assist deaf men and women, within a relatively comfortable online environment, to identify potential matching partners and provides dating opportunities. The online site allows its members to create a personal profile and to identify a suitable partner for dating based on their personal preferences. It serves as a source for creating healthy relationships ranging from companionship to friendship, romance to marriage [11].

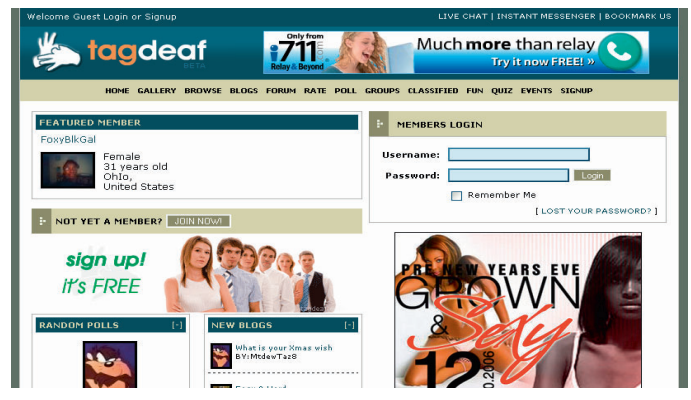

Figure 3. Tag Deaf

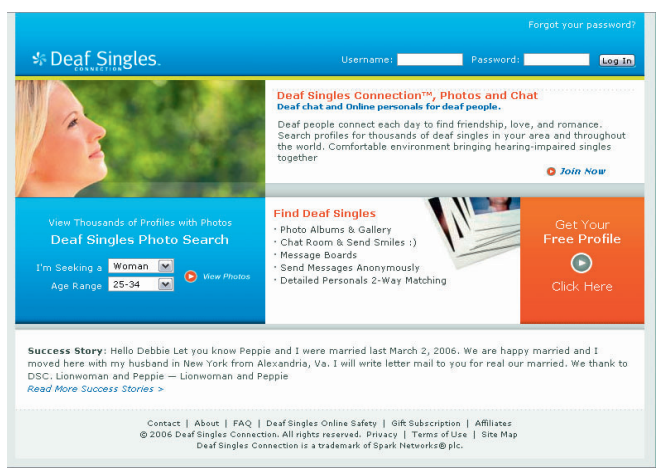

Figure 4. Deaf Singles Connection

In general, many deaf people also join other popular communities such as Friendster, MySpace and Facebook, but a majority of them would like to opt for joining their own niche, online communities in order to serve and share their interests on deaf issues and concerns. The following diagram (Table 1) compares, illustrates and summarizes the features offered by current online communities:

TABLE I. A

COMPARISON OF FEATURES FOR EXISTING ONLINE COMMUNITIES.

\begin{tabular}{|c|c|c|c|c|c|c|c|c|c|c|c|c|}
\hline \multicolumn{13}{|c|}{ Comparison Charts for Existing Online Communities } \\
\hline \multicolumn{13}{|c|}{ Features } \\
\hline Online Community & Forum & IM Chat & Profile & Blog & News & Email & Fun & Group & Gallery & Quiz & $\log \operatorname{In}$ & Others \\
\hline Deaf Online Forum & - & & - & e & - & ? & & & 0 & 0 & 0 & $\begin{array}{l}\text { Calcndar, Discussion, } \\
\text { Scarch, Avatar }\end{array}$ \\
\hline All Deaf Forum & 0 & & 0 & & - & - & 0 & & - & & 0 & $\begin{array}{l}\text { Calcndar, Disscussion, Avatata } \\
\text { Advertisenent, Search, }\end{array}$ \\
\hline TagDeaf & 웅 & 웅 & 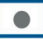 & 0 & 인 & 웅 & 0 & 0 & 웅 & 9 & 0 & $\begin{array}{l}\text { Bulletin Boards, Shoutbox, } \\
\text { Polls, Classifieds }\end{array}$ \\
\hline Deaf Singles Connection & 0 & 0 & 0 & & & - & 0 & 0 & 0 & & 0 & $\begin{array}{l}\text { Browsing, E-Cards, Private } \\
\text { Mailbox, Matching, Search }\end{array}$ \\
\hline hiype! & 0 & & & & 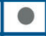 & & 0 & 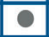 & & & & $\begin{array}{l}\text { Job Hunt, Polls, GuessBook, } \\
\text { Message box }\end{array}$ \\
\hline Friendster & & & 0 & 0 & 우 & 0 & 0 & 0 & 으 & & 은 & \begin{tabular}{|} 
Classified, Vidoo, Search, \\
Shoutout, Bookmarks
\end{tabular} \\
\hline MySpace & & 은 & - & 0 & 우 & 0 & 0 & 은 & 운 & & 운 & $\begin{array}{l}\text { Classified, Video, Search, } \\
\text { Comety,Music, Film }\end{array}$ \\
\hline Facebook & 0 & & 은 & 0 & 는 & 은 & 0 & 우 & 은 & & 0 & \begin{tabular}{|l} 
lobs, Music, Movies, Books, \\
Bulletin Boards
\end{tabular} \\
\hline Classmates.com & & & O & & 0 & 9 & 0 & 0 & ? & & 0 & \begin{tabular}{|l} 
School group, build website \\
tools, event reunions
\end{tabular} \\
\hline
\end{tabular}

\section{Design Process Model and Design Methods}

\section{A. The Design Process Model}

In the Interface Design department, we adopted and integrated a modified Pugh's Design Process Model [12] in our user-centered design and development process, namely User Interface Design Process Model. The iterative design process was conducted within the context of multimedia and content design. The process was developed to cater, adhere and consist of the user-centered and interface design elements. Human factors methods and design tools were fused together to provide a holistic design approach as described in the diagram below (Fig. 5):

Based on the fundamental Pugh's Design Process Model [12], the design studies were conducted at the concept design stage of the model. Specific human factors techniques and design development tools were identified and applied at this stage of the design process model accordingly. Although the DHearT mobile community is a non-physical design and development activity, the design and human factors techniques that are usually applied on a physical design are still relevant and usable. For example, market research, presentation techniques, user profiling, user observation, design specifications, and mood board. 
The User Interface Design Process Model (Fig. 6) lists the simplified iterative design process, which was utilized in the design and development of DHearT. It highlights the design techniques and human factors activities under the market research, mood board, and design narration.

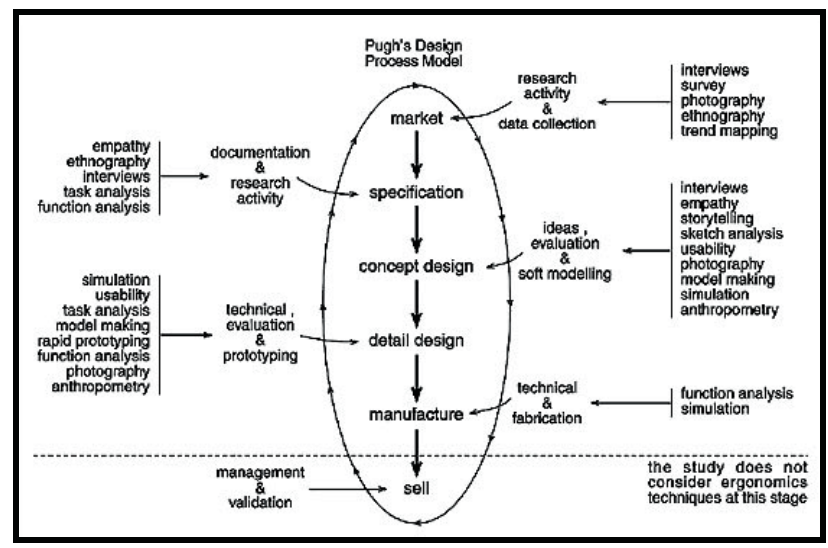

Figure 5. A modified Pugh's Design Process Model adopted for usercentered interface design

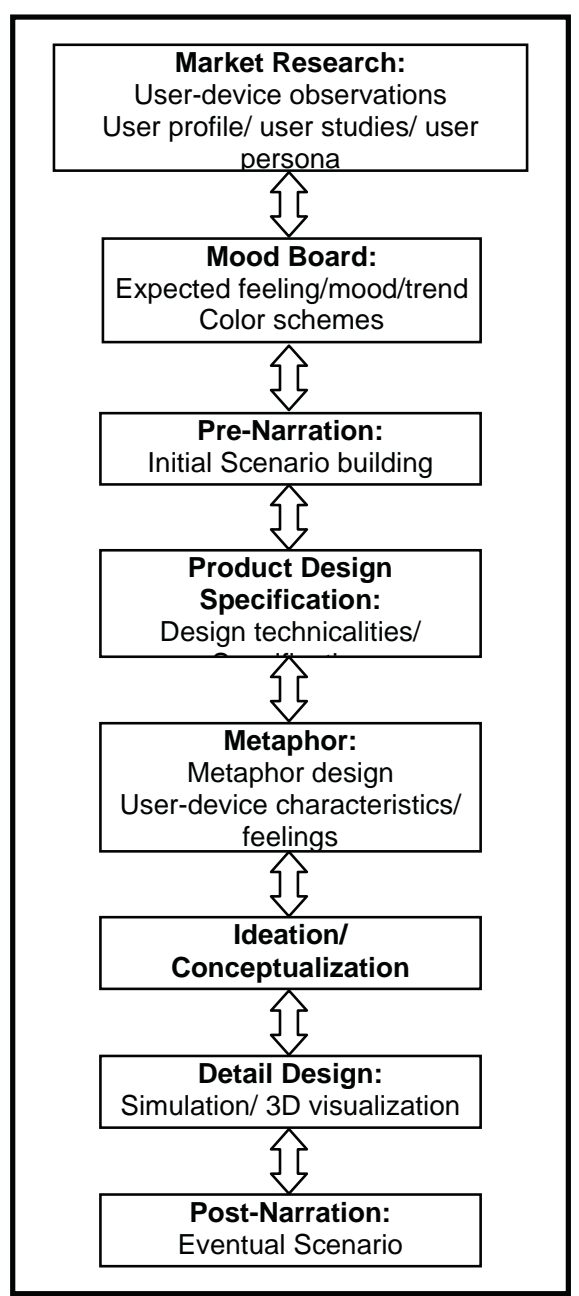

Figure 6. A simplified User Interface Design Process Model diagram deriving from Pugh's Design Process Model showing the design tools and human factors techniques deployed in the development of DHearT

\section{IMPLEMENTATION OF DESIGN TOOLS AND APPLIED HUMAN FACTORS TECHNIQUES}

\section{A. User Studies}

The research team consisted of 2 designers and 2 human factors specialists, of which an interface designer is also a hearing impaired person. A series of user studies and fieldwork were conducted in two phases from July 2006 until January 2007. A user survey was conducted in the first phase from July till September 2006 to investigate the feasibility study and acceptance level of 3G mobile technology among the Malaysian deaf community [2]. In the second phase, we conducted user studies via in-depth interviews and observations from October 2006 till January 2007. The studies and fieldwork was to elicit user requirements for mobile communities among the local deaf.

\section{B. User Profiling}

Two main target groups were identified as our potential users in this study. They were the local deaf community with ages from 16 to 45 years old, and also the hearing groups, basically comprised of parents and friends of the deaf and those who are interested to communicate with the deaf. In order for us to reach the right community segment, we approached local NGOs and organizations such as the Malaysian Federation of the Deaf (MFD), Majudiri Y Foundation for the Deaf (MYF), Kuala Lumpur YMCA Deaf Club, and Kuala Lumpur Society of the Deaf.

In general, a majority of the local deaf population do not receive advanced education and adequate skills as required for them to survive in their daily employment. At this moment, there is no ruling enforced or being in effect with regards to the Disability Employment Act in local corporate and industries. Hence, the deaf basically find difficulty in securing suitable career prospects compared to hearing people. Such issues point to the need for an interface proving a medium for both deaf and hearing communities to interact and communicate with one another.

Below is a summary of the user profiles for the deaf community:

$$
\text { - Target users: }
$$

16 - 45 years old; female and male; deaf or partially deaf.

\section{- $\quad$ Educational background:}

Around $68 \%$ of Malaysian deaf teenagers and adults understand Bahasa Malaysia, and 32\% understand English in an acceptable advanced level equivalent to the British "Ordinary" (O) level in the local education system. The low percentage of English comprehension is due to the widespread use of Kod Tangan Bahasa Melayu and Manual Sign Language in Bahasa Malaysia, which is mainly taught as the main command of "language" to the deaf under the special education system in the country. In the past few years, the local deaf community started to improve their English with the support of e-Pekak Information Communication and Technology (ICT) portal. The educational background for the local deaf has tremendously increased to diploma and degree level as compared to the past with non-degree qualifications. Most deaf students and teenagers, who study in special schools are exposed to vocational training, including problem 
solving, decision making and also different living skills in life.

- $\quad$ Learning styles:

Basically deaf people are visual and verbal (text-based) learners.

\section{- Occupation:}

Social workers (working at NGOs), IT administrators, accountants, designers, clerical workers, administrative staff and students.

$$
\text { - Lifestyle: }
$$

Like other hearing people, the deaf have their own hobbies such as shopping, sports, social activities and so forth. They would like to spend time doing chatting via instant messengers, browsing Internet, playing games, watching movies and also texting via mobile phones.

- $\quad$ Attitude towards computer, Internet, and mobile phone experience:

Novice, experienced or experts.

\section{- Computer Experience:}

Some deaf use personal computers daily, or at least twice a week. Some are conversant with graphical software such as Microsoft Powerpoint, Adode Photoshop and Adobe Illustrator. Basically, they are able to use the Internet for browsing, information retrieval, chatting via instant messengers, and reading blogs. Some prefer to use webcam to conduct 'sign' language activities with their family and friends for better communication depending on the available network connectivity.

- $\quad$ Problems the deaf face in their daily lives:

The command of English for the deaf is generally of a lower standard compared to the hearing groups. This affects their understanding of written language. This scenario is obvious when the deaf find difficulties to understand the verbal expressions (e.g. uuh, ahh, yup, err..., etc.) and also short form in SMS (e.g. '2day' stands for 'today', 'tmr' means 'tomorrow', etc.)

The deaf and hearing impaired find that the written message in chat and messenger software programs are 'boring and dull', where they are required to type long sentences. Inevitably, this takes up a lot of time in typing. It is also noted that a chat tool which allows for 'signing' instantly is preferred. Although the deaf generally like emoticons (e.g. smiley faces) in the instant messengers, they sometimes find it difficult to comprehend the meaning of the emoticon without an explicit description of it and also to be explained in sign language. The deaf also find that there is a need for a dictionary facility to pose as a communication aid to explaining a situation that the deaf person may be feeling. Again, this poses the need for an intermediary device or aid to help the deaf communicate with others using sign languages directly via mobile services and this is not available yet in the country.

\section{User Persona}

The user personas were the results and insights derived from user studies which we then translated into personas. Cooper [13] outlined 'personae or personas as fictitious characters that are created to represent the different user types within a targeted demographic (profile) that might use a site or product.' Personas are given characteristics and are assumed to be in particular environments based on known users' requirements so that these elements can be taken into consideration when creating scenarios for conceptualizing a site. The design process utilized in this project included the creation of personas in an attempt to assist the designers to identify and quantify the needs of the intended users. In this study, we created 2 main categories of user personas representing the deaf and hearing community. Due to the length of the paper, we illustrated one example of user persona for a working deaf user (see Table 2).

TABLE II.

A USER PERSONA FOR A WORKING DEAF.

Name: Aqlam Jafri
Age: 35
Gender: Male
Occupation: Information System
Admin
Nationality: Malaysia
Education History:

He was the first deaf Malaysian student who graduated from Gallaudet University, Washington DC, USA. He had a Bachelor Degree in Computer Information Systems. He previously studied in the special primary school and hearing secondary school in Johor Bahru before Gallaudent University. He is skillful in Bahasa Malaysia sign language and also American Sign Language. Sometimes he will fly to USA to learn new sign language and receive training in assistive technologies. As return, he will transcend the training across to the local deaf community. He is currently the President of KLSD (Kuala Lumpur Society Deaf).

\section{Computer Experiences:}

$\mathrm{He}$ is skillful in using a computer. He is good at teaching and providing training of ICT to the deaf students and active in helping the local deaf community. Apart from ICT, Aqlam also teaches the deaf about soft skills like leadership in order for them to solve problems in their daily lives. He likes to chat with his deaf friends using the yahoo and msn messenger. However, he finds limitation of using the instant messenger, and would opt for something more specific for the local deaf.

\section{Lifestyles:}

He loves to spend his time with his daughter and wife every weekend. He is active in social work and contributes to the local deaf community. He has a high commitment to his work and plays an important role in local NGOs and deaf clubs in creating deaf culture awareness campaign.

The problem Aqlam faced while communicating with the deaf and hearing people:

Aqlam sometimes finds difficulty in using his mobile phone, especially sending SMS to his deaf friends. For instance, he has a $3 \mathrm{G}$ mobile phone but most of the deaf people do not have $3 \mathrm{G}$ mobile phones. He prefers to see a fellow deaf's facial expression whilst he himself is 'signing' through the video phone. The SMS feature is very limited for the deaf community with less accurate expressions. He communicates with his hearing friends using Yahoo! Messenger, especially for meetings and discussing projects. He hopes to utilize the video features in the chat room for the benefit of the deaf community. 


\section{Metaphor Studies}

Metaphors have been widely used in the design discipline to inspire and to help explain concepts. In the interface design process model, we use and apply metaphor to associate the primary object (metaphor) into a new conceptual design. The concept of a metaphor is used in the design process to assist designers to contextualize and to provide inspiration in conceptualizing designs. In this project, a ferret was chosen to represent the DHearT mobile community. Ferret is a small, weasellike animal; a sociable creature in its class and has characteristics of being playful.

This includes liking to squirm and jump, and greatly enjoy being surrounded by humans. The personality of a ferret varies; in general they are happy, joyful, inquisitive, bouncy, fearless, active, quiet, clean, humorous, charming, playful and generally entertaining to watch. A ferret needs several hours of free time a day and thrives on a lot of human interaction. This includes praise, cuddling, love and a dash of discipline. Ferrets live to play and prefer simple toys such as ping pong (table tennis), golf balls, old socks, paper bags and others. [14]. Due to its characteristic, a ferret was chosen as a metaphor because the deaf community naturally loves to interact with others. The deaf people are generally lovely, peaceful, sociable, and needs friendship like other hearing people as found from the characteristics of a ferret.

\section{E. Icon Design}

The metaphor of a ferret used in this project was implicitly transformed into a series of icons. This includes simplified icon designs representing functional attributes such as profile, deaf news, camera, bulletin board, blogs, message box, gallery photo, jobs, horoscopes, games, fun box, sign ABC, sign 123, quiz, my profile, news, sign builder, sign learning, basic sign, trash, upload blog and upload photo (Fig. 7).

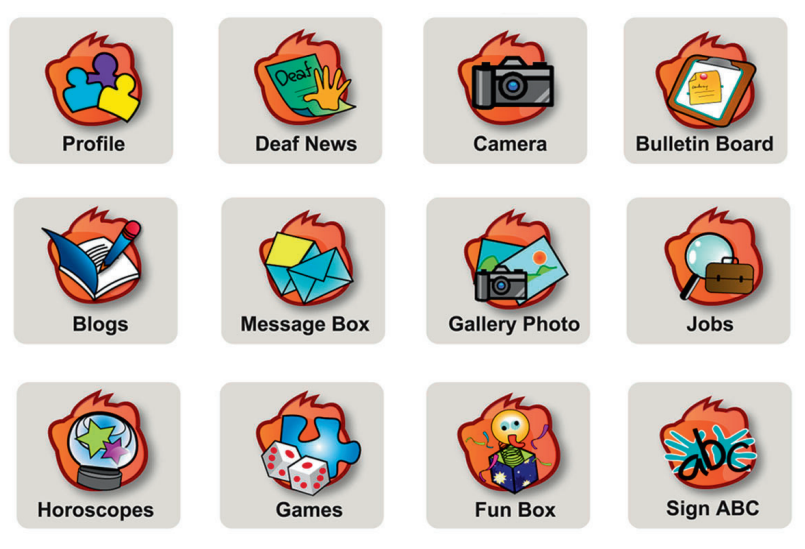

Figure 7. Icon designs developed for DHearT mobile communities.

\section{F. Menu Design}

The menu for mobile phones was designed for its intended users of ages 15 and above. The background color scheme is beige or pale yellowish brown to allow the icons and text to stand out clearer. The main menu offers a 2-second animated "cog” when accessed, followed by the appearance of the avatar greeting the user. Users use the left-right keys on the mobile phone to navigate and the middle button to "accept" or "select" items. Tool tips appear on icons that are highlighted during the user's navigation, and the avatar was programmed to show the appropriate sign language at the same time.

We designed a main menu with six categories to represent the main functions of the community. This includes "jobs", "fun box", "blog”, "news", "sign learning” and "profile” (Fig. 8). Each menu category was designed to have less than 5 levels of hierarchy down. This was to ensure that users will not get "lost" within menu categories and also to avoid nesting and intercategory usage. Below are some screen shots of the menu categories and background for DHearT mobile community (Fig. 8 - 11):

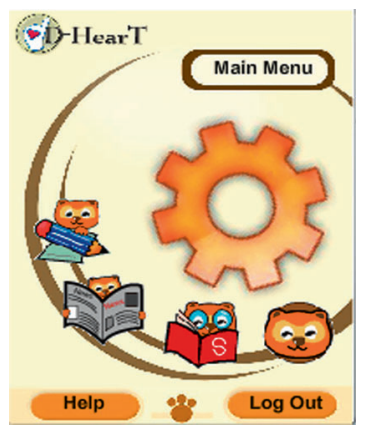

Figure 8. Main menu loading with animated cog.

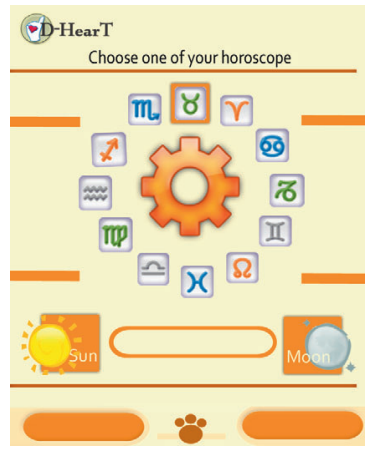

Figure 10. Menu design for 'Horoscope'.

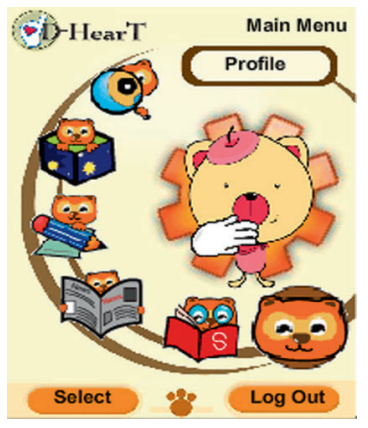

Figure 9. Main menu with 'Profile' selected by default.

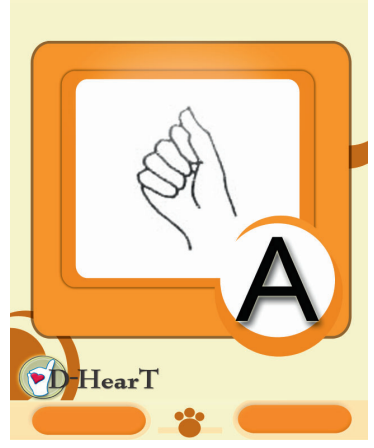

Figure 11. Aphabet 'A' in sign language.

\section{User Testing the Prototype}

We conducted initial user trials with 10 users (5 deaf and 5 hearing) to gather preliminary user feedback on the DHearT prototypes. These users are the intended users as outlined by the project's user profile, narration and observation studies.

The user trials were conducted in two phases. The first phase of the user trial was conducted using a simulated DHearT prototype on the personal computer (PC) as shown in Fig. 12. This was to gain first-hand information from the intended users' about the overall look and feel of the proposed mobile community. These initial evaluations were also meant to help filter out program bugs, to determine design flaws and to help troubleshoot potential networking issues in the simulation. The mode of interaction for the prototype shown on the PC was the computer mouse and keyboard. 


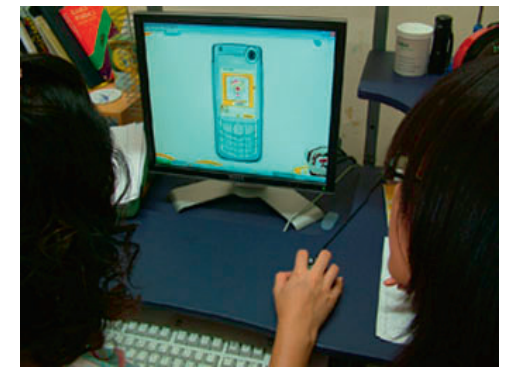

Figure 12. First phase of DHearT user evaluations were carried out as simulations on a PC.

The second phase of user evaluations was conducted for DHearT on the Symbian S60 series of Nokia N70 and N6630 mobile phone models (Fig. 13). Users were provided with a set of tasks and the time was taken down to complete the given tasks. The main mode of interaction in this phase of trials is the phone keypad.

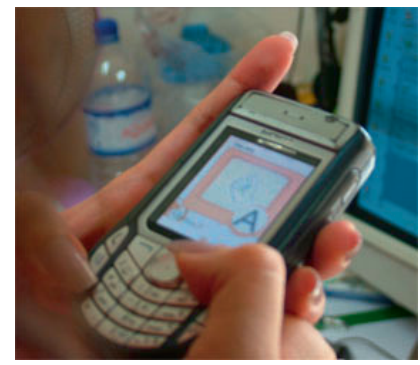

Figure 13. Second phase of user-device evaluations on mobile phones.

\section{DESIGN IMPLICATIONS: DISCUSSION}

\section{A. Design Process and Applied Techniques}

The User Interface Design Process Model, deriving from Pugh's Design Process Model, indicates a more simplified and practical approach for a design team in the product development process. Several design tools and human factors techniques were employed by the interface designers for the implementation of DHearT mobile community project. For instance, observation tools and techniques such as photography, video recording, notetaking, and audio recording were used by the design team to solicit user-device requirements at the market research stage. We conducted several brainstorming sessions to produce possible user-device needs, directions, trends and preferences to trigger conceptual ideas for a mobile community design project. The data we gathered helped to define targeted user profiles and market trends.

While developing possible mood boards and conceptualizing directions for trend, we found the initial knowledge of user-device information gathered from the market research seeping into the design activity to be very helpful. This immediately assisted us in the selection of color schemes and user trends prior to generating concept ideations. The information also provided more depth for the development of the pre-narration user scenarios. The trickle of preliminary user-device information provided us a notion of design direction, helped bring our ideas together, and assisted in trying our design and development activity into a more focused process.

\section{B. Designing the Interfaces}

In terms of interface design aspect, the overall menu and icon design had to be created to cater for existing low color compatibility of mobile phones. Designing a mobile community for a small screen size on a mobile phone is always a challenging process for the designer and developer community. To counter balance the technical constraints, selected color scheme settings had to cater for TFT (thin film transistor) screen types that support 65,000 to 256,000 colors. Hence, the icons, animated avatar, and overall menu design had to conform to these settings. We had to scale down the color settings while designing and scripting from PC to suit for the mobile platform. We also considered screen adaptation for two screen formats (portraits and landscape) on different mobile phones. At the moment, we designed DHearT based on N70 and N6630 mobile phone templates, with the physical and pixel screen size of 35mm x 41mm (176 x 208 px). However, the technical challenge we faced was to ensure the design template suits any screen size of different phone models. In the future, we will employ using mobile trans-coding facility to accommodate and optimize the best screen adaptation for different mobile devices.

The menu was mainly designed for single-handed operation; left-right function keys and the middle button. Where typing is required, for instance when users start typing a message, or when playing the integrated game and operating the camera, the users will need both hands to operate the online community program.

\section{Prototype Testing and User Feedback}

We designed and developed DHearT on a PC platform. Although the intention is for the project to be implemented on a mobile device, the software development kit (SDK) and peripheral activities involved such as scripting, and screen management may not work well on the device platform. We found that there were many incompatibility issues for user testing on a mobile phone.

The two initial phases of PC and user-mobile phone evaluations were very useful for the design team to reconsider scripting approaches and interface design. Simulations on the PC involving elements such as color depth, gradient, animation frames per second (fps), keypad press (mouse click), and appearances of tool tips were all well supported. However, these elements had to be re-configured to meet the lower processor specifications and display color depth on mobile phones.

With the understanding of technical limitations of making DHearT a fully functional prototype, our aim of user testing was to gather user feedback on the overall 'look and feel' of the interface design. Therefore, we adopted a 'quick-and-dirty' qualitative approach to gather user feedback to scan through the interface design issues. For instance, we adopted protocol analysis to encourage the users to verbally 'express' their thinking by 'walkingthrough' the menu designs.

To summarize the user evaluation sessions, the deaf users generally liked the concept of DHearT to cater for their needs while on the move. The young deaf users (1622 years old) somehow like the avatar and icons. However, they prefer to customize their avatar with other pets' options instead of merely ferret's logo. They also expressed the sign language feature would enhance the 
learning process with the support of the video feature. However, they highlighted 3G camera phones will help streamline the communication process by 'signing' via the video features with their friends. For the 'hearing' groups, they generally liked DHearT as a novel idea in Malaysia. This was due to the fact that there are currently no other existing technologies available in the local market to bridge the gap between the hearing and the deaf community. The 'sign language' texting was fun to use and learn the language, but they thought it was somehow slow in communicating with the deaf. Overall, given the genuine conceptual ideas, the users were excited and looking forward to see a full functional DHearT mobile community project available for the future market.

\section{VIII.CONCLUSION}

In this paper, we presented the user-centered design and adopted the User Interface Design Process Model for DHearT, a creation of a mobile-based community for the deaf and hearing groups in Malaysia. We have reviewed relevant, existing internet-based communities, and also outlined the design and development of the DHearT mobile-based community. In addition, we have illustrated the adoption and application of Interface Design Process Model with the supporting design tools and human factors techniques.

Due to the length of the paper, we only discuss several design methods for DHearT mobile community. We plan to discuss and scrutinize more detailed work on the application of the design process in a more comprehensive manner in the future. Given the scenarios of technical constraints, the first two phases of user feedback were not able to cover a 'functional' working prototype for some task scenarios. Thus, this somehow has discounted the overall functionality and usability of DHearT during the user trials. Thus, more rigorous usability testing will be employed to study the attributes of effectiveness, efficiency and satisfaction on the mobile community design in the future.

Future studies will also involve working together with the local mobile operators and NGOs for further enhancement and development of DHearT mobile community before launching to the masses. This move will provide us the opportunity to produce a national framework and scaffolding guidelines, and for addressing the digital divide between the deaf and hearing communities.

\section{ACKNOWLEDGMENT}

We would like to express our gratitude to Selina S.P. Ooi and Siao Ying Chean for their contributions of DHearT design project mentioned in the text. In addition, we are also indebted to our collaborating partner, Telenor Research and Innovation Centre Asia Pacific (TRICAP) for their input and feedback during the design assessment. Many thanks to the NGOs (Majudiri Y Foundation for the Deaf and Malaysian Federation of the Deaf) for their assistance, users who participated in the interview and evaluation of prototype of DHearT mobile community.

\section{REFERENCES}

[1] Malaysian Communications and Multimedia Commission (MCMC), Q4 2007 Communications \& Multimedia Selected Facts and Figures, Retrieved December 15, 2007 from http://www.mcmc.gov.my/facts_figures/stats/index.asp

[2] C.Y. Wong and C.W. Khong, "Constructing a UTAUT Model of 3G Mobile Technologies for the Hearing Impaired Community," in Proceedings of the Human-Computer Interaction International Conference, Beijing, China, July 2007, C. STEPHANIDIS, Eds. Lecture Notes of Computer Science (LNCS 4556), Springer, DVD-ROM, 2007.

[3] Malaysia Council For Rehabilitation, A Review on the decade of disabled persons 1993 - 2002: A Malaysian NGO Perspective, 2002. Retrieved August 30, 2006 from http://www.dinf.ne.jp/doc/english/intl/02rnn/malaysia_e.html

[4] World Wide Web Consortium (W3C), "How People with Disabilities Use the Web,” Working-group Internal Draft, May 5, 2005. Retrieved on April 10, 2006 from http://www.w3.org/WAI/EO/Drafts/PWD-Use-Web/

[5] L. Lim, S.Woo, A. Chong, K.Schmidt, G.W. Yip, and K.W. Ho, Understanding Deaf Culture: Malaysian Perspectives. Malaysia: Majudiri Y. Foundation for the Deaf, 2006.

[6] C. Padden and T. Humphries, Deaf in America: Voices from a culture. Cambridge, MA: Harvard University Press, 1988.

[7] R. Southwick, "Deaf culture and Ministry: Consider and Ponder these thins in your heart," 2003. Retrieve on December 6, 2006 on http://frontpage.erie.net/dwm/articles.html

[8] DEAF ONLINE, “What is DOL?” 2004. Retrieved on December 11, 2006 from http://www.deafonline2.com/forums/index.php?act=home

[9] ALL DEAF, “Deaf forum for all deaf people,” 2002. Retrieved on December 10, 2006 from http://www.alldeaf.com

[10] TAG DEAF, “Deaf Social Network,” 2006. Retrieved on December 10, 2006 from http://www.tagdeaf.com

[11] DEAF SINGLES. "Deaf Singles Connection," 2006. Retrieved on December 9, 2006 from http://www.deafsinglesconnection.com

[12] S. Pugh, Total Design, Integrated Methods for Successful Product Engineering. US: Addison-Wesley, 1990.

[13] A. Cooper, The Inmates are running asylum. US: SAMS, 1999.

[14] C.O. Donnel, "An Introduction of Ferret Care. Ferrets 101," 2006. Retrieved on December 3, 2006 on http://www.fuzies.com/FARI/fari.htm

\section{AUTHORS}

Chui Yin Wong (cywong@mmu.edu.my).is currently the Industrial R\&D Liaison cum Lecturer for Interface Design Department, Faculty of Creative Multimedia, Multimedia University Malaysia, 63100 Cyberjaya, Malaysia.

Chee Weng Khong (cwkhong@mmu.edu.my) is currently the Associate Dean (R\&D and Commercialization) for the Faculty of Creative Multimedia. He is also the Head of Interface Design Department, Faculty of Creative Multimedia, Multimedia University Malaysia, 63100 Cyberjaya, Malaysia.

Harold Thwaites (harold.thwaites@mmu.edu.my) is the Dean of the Faculty of Creative Multimedia, Multimedia University Malaysia, 63100 Cyberjaya, Malaysia.

This article was modified from a presentation at the 21st Symposium on Human Factors in Telecommunication (HFT 2008) in Kuala Lumpur, Malaysia, March 2008. Manuscript received 01 September 2008. Published as submitted by the authors. 